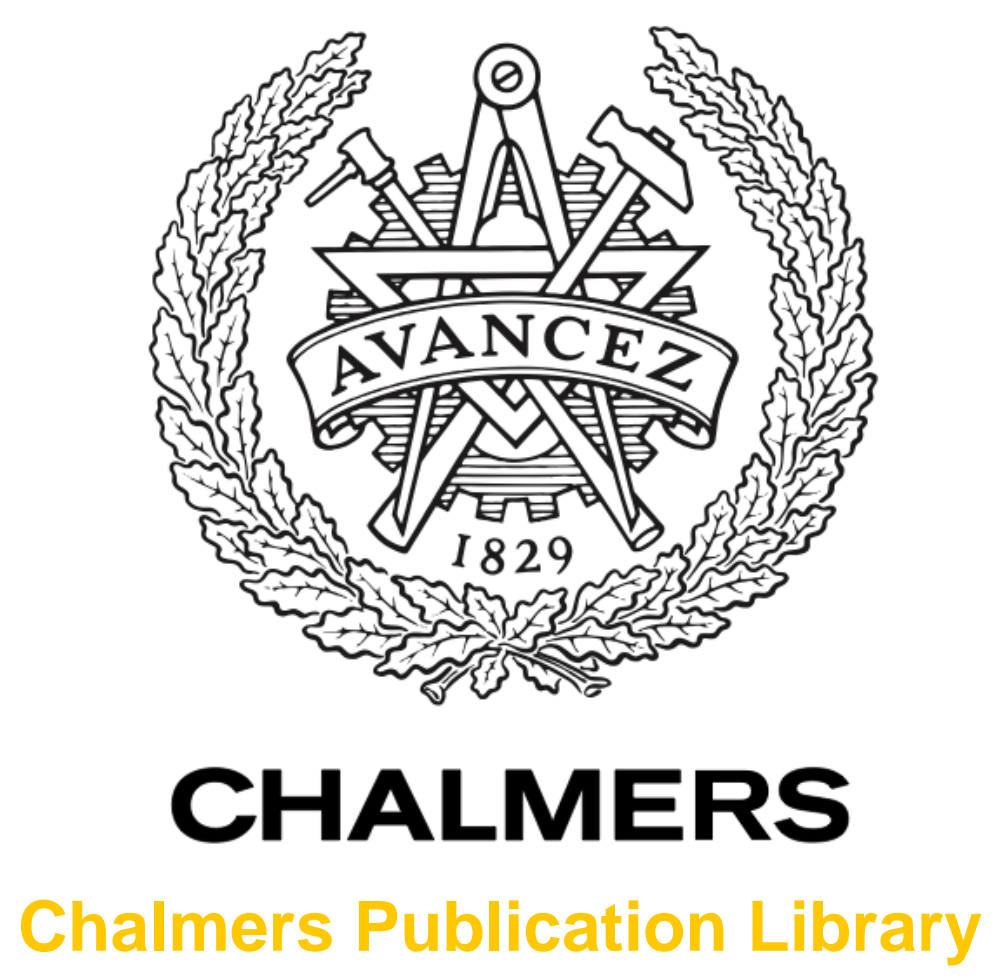

Freeway ramp metering: an LPV set theoretical analysis

This document has been downloaded from Chalmers Publication Library (CPL). It is the author's version of a work that was accepted for publication in:

IEEE American Control Conference, San Francisco, CA, USA, June 29-July 01, 2011 (ISSN: 07431619)

Citation for the published paper:

Luspay, T. ; Kulcsar, B. ; Peni, T. (2011) "Freeway ramp metering: an LPV set theoretical analysis". IEEE American Control Conference, San Francisco, CA, USA, June 29-July 01, 2011 pp. 733-738.

Downloaded from: http://publications.lib.chalmers.se/publication/137026

Notice: Changes introduced as a result of publishing processes such as copy-editing and formatting may not be reflected in this document. For a definitive version of this work, please refer to the published source. Please note that access to the published version might require a subscription. 


\title{
Freeway ramp metering: an LPV set theoretical analysis
}

\author{
Tamás Luspay, Balázs Kulcsár, Tamás Péni and István Varga
}

\begin{abstract}
The paper contributes to the analysis of freeway traffic flow dynamics by set theoretic methods.

First, the macroscopic, non-linear and second-order model of freeway traffic flow dynamics is transformed to an equivalent and quasi Linear Parameter Varying (LPV) representation by steady-state centering and state variable factorization. Second, a polytopic LPV model form is obtained from the quasi model reformulation. The latter polytopic LPV form is then used as a basis for the computation and analysis of disturbance invariant sets. This framework is able to characterize constrained sets of states which can be reached by pure ramp metering control input signals. Furthermore, these sets become invariant to other measured and unmeasured disturbance inputs.

The application of disturbance invariant set theory provides an analytical tool for constrained freeway ramp metering describing the set of states being invariant under the system dynamics, measured disturbance and other physical constraints regardless to the value unmeasured disturbance signal.

The proposed idea is fully based on the analysis of the (transformed) non-linear macroscopic system and aims at filling the gap between the traffic modeling and quantitative freeway ramp metering.
\end{abstract}

Index Terms-Set theory, ramp metering, polytopic systems, linear parameter varying representations

\section{INTRODUCTION}

The aim of almost all traffic control algorithm is to ensure the best reachable network throughput without the extension of the available infrastructure. Among others, freeway ramp metering is considered as the most common freeway traffic control measure. It has been in the focus of transportation engineers since 1980, and many solutions have been developed, published and implemented [1].

Ramp metering algorithms can be divided into two main groups; static or fix algorithms and dynamic or traffic responsive methods. Static algorithms can be designed on the basis of batch mode traffic measurements. The largest drawback of such algorithms is their incapability to address to actual traffic conditions. This drawback can be overcome by introducing actual traffic measurements dependent methods.

The question of designing traffic responsive ramp metering is always challenging, since one has to clearly know the effect of ramp metering in the given traffic condition. This knowledge necessitates a proper dynamical model of the underlaying process. Second-order macroscopic freeway

T. Luspay, T. Péni and I. Varga are with Systems and Control Laboratory, Computer and Automation Research Institute, Hungarian Academy of Sciences, H-1111 Kende u. 14-17. Budapest, Hungary \{tluspay; ivarga\}esztaki.hu, ptescl.sztaki.hu.

B. Kulcsár is with Department of Signals and Systems, Chalmers University of Technology, SE-412 96, Gothenburg, Sweden kulcsar@chalmers.se. models are possible candidates to serve as a basis of such a design, due to their ability to accurately reproduce traffic phenomena.

These models describe the dynamical evolution of traffic variables with non-linear differentia equations, implying the need of non-linear control synthesis techniques. Moreover, the control problem is subjected to hard physical constraints. Due to the lack of systematic non-linear constrained control design, existing ramp metering solutions are either use linearization techniques [2], or other (approximate) numerical methods [3].

Although these algorithms are known in the literature, no systematic analytic method of the closed-loop problem have been presented yet, mostly because of its computational complexity and lack of algorithmic solutions. The aim of the paper is to provide a tractable, model-based analysis framework for freeway ramp metering. Therefore, the original and nonlinear second-order macroscopic model has been chosen and transformed into a polytopic form. The resulting structure preserves the accuracy of the original model, but in a compact form. This representation makes the application of advanced analysis and control methods available for freeway traffic systems. In the paper set-theoretic tools are used for studying the local ramp metering.

The paper is organized as follows. After the Introduction section, the basic traffic notations are introduced and the ramp metering problem is stated (Section II). The model transformation and the description of the polytopic model is given in Section III. A set-theoretic analysis method for the obtained polytopic structure is discussed in Section IV. Numerical example is given in Section V to illustrate the proposed method.

\section{PROBLEM STATEMENT}

A possible method to model the dynamics of freeway traffic systems is to consider the macroscopic approach, based on the fundamental analogy between streaming fluids and interacting vehicular motion. In these concepts, the key idea is to neglect individual vehicle motions and only use aggregated and traffic related variables such as traffic density $(\rho,[\mathrm{veh} / \mathrm{km}])$, space-mean speed $(v,[\mathrm{~km} / \mathrm{h}])$ and traffic flow $(q,[\mathrm{veh} / \mathrm{h}])$. Through the paper a second-order macroscopic model is used to represent the dynamics of freeway traffic flows [4]. The model equations for an arbitrary stretch of freeway with an on-ramp connection (Figure 1.) can be 


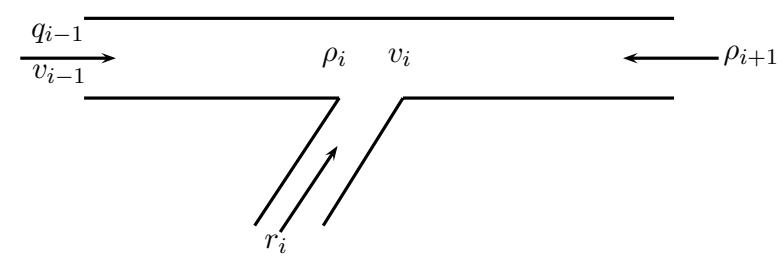

Fig. 1. Illustration of a freeway segment

formulated in discrete time and space as follows:

$$
\begin{aligned}
\rho_{i}(k+1) & =\rho_{i}(k)+\frac{T}{\Delta_{i} n}\left[q_{i-1}(k)-q_{i}(k)+r_{i}(k)\right], \\
v_{i}(k+1)= & v_{i}(k)+\frac{T}{\tau}\left[V\left(\rho_{i}(k)\right)-v_{i}(k)\right]+ \\
+ & \frac{T}{\Delta_{i}} v_{i}(k)\left[v_{i-1}(k)-v_{i}(k)\right] \\
& -\frac{v}{\tau} \frac{T}{\Delta_{i}} \frac{\rho_{i+1}(k)-\rho_{i}(k)}{\rho_{i}(k)+\kappa}-\frac{\delta T}{\Delta_{i} n} \frac{r_{i}(k) v_{i}(k)}{\rho_{i}(k)+\kappa}, \\
V\left(\rho_{i}(k)\right)= & v_{f} \exp \left[-\frac{1}{a}\left(\frac{\rho_{i}(k)}{\rho_{c r}}\right)^{a}\right], \\
q_{i}(k)= & \rho_{i}(k) \cdot v_{i}(k) \cdot n .
\end{aligned}
$$

The subscript $i$ indicates a small spatial stretch of $n$-lane freeway with length $\Delta_{i}$. In the sequel, we refer to such a spatial freeway stretch as a segment. Traffic variables are discretized in time using time step $T$. Segment's dynamics are described with the help of two traffic variables; $\rho_{i}$ and $v_{i}$. On-ramp volume $r_{i},[\mathrm{veh} / \mathrm{h}]$ is considered as input variable to assign freely based on the actual traffic conditions and control objective [3]. This control input is subjected to hard physical constraints; $r_{\min } \leq r_{i} \leq r_{\max }$. Traffic variables and conditions influencing the segment's variables are partially modeled as forward and partially as backward effects. The upstream segment $(i-1)$ is connected through its boundary variables such as traffic flow $q_{i-1}$ and space-mean speed $v_{i-1}$ to the segment $(i)$, while downstream segment $(i+1)$ has a backward effect through its density $\rho_{i+1}$ variable. In freeway traffic systems, it is common to measure upstream variables with loop detectors, while downstream variable is usually not measured [6]. Model parameters $\tau, v, \kappa, \delta, a, v_{f}, \rho_{c r}$ can be determined by various methods e.g. [6], [7].

Equation (3) is known as a fundamental relationship, reflecting how the space-mean speed decreases in function of the increasing traffic density. The parameters of eq. (3) can be determined by non-linear fitting to detector measurements (see Section V), [6], [7]. By using the relationship in eq. (3) together with the formula of eq. (4), the fundamental diagram is yielded characterizing the average throughput of the segment as a function of the traffic density. Based on the fundamental diagram and traffic measurements, it is indicated that freeway segment has a maximal capacity, which coincides with the density and speed values called critical $\left(\rho_{c r}, v_{c r}\right)$. Once the traffic flow reaches the regime behind $\rho_{c r}$, it becomes congested throughput start to decrease. One of the major aim of traffic control is to avoid such capacity fall-off. Also, it is obvious that traffic control measures are constrained and limited and consequently not every traffic conditions can be handled adequately. The ultimate goal of the paper is to characterize quantitative model-related regions where freeway traffic control, more precisely ramp metering, is still efficient and maximal capacity can be achieved.

\section{POLYTOPIC MODEL}

Non-linear differentia equations in eqs. (1)-(4) are able to reproduce macroscopic traffic phenomena. The disadvantage of the representation from the point of analysis methods is its complexity and non-linear nature. To handle nonlinearity and decrease computational load and algorithmic complexity an equivalent form has been elaborated for modeling [5], identification [7] and for (control related set theoretic) analysis in the sequel.

The model transformation suitable for such an analysis can be derived in two main steps, as proposed in the paper. First, reformulation of the non-linear dynamics into a general Linear Parameter Varying (LPV) form (III-A) and transformation of the obtained LPV model into a polytopic structure using Tensor Product (TP) method (III-B).

\section{A. LPV formulation of the non-linear dynamics}

Reformulation of the non-linear dynamics into affine, discrete time Linear Parameter Varying form can be derived by [5]. Hereunder follows the slightly modified major derivation steps.

1) Determination of steady state conditions. The discretetime steady state condition $\left(x(k+1)=x(k)=x^{*}\right)$ is applied for the dynamical equations (1)-(2). The resulting two non-linear algebraic equations are described by six unknown variables: $q_{i-1}^{*}, v_{i-1}^{*}, \rho_{i}^{*}, v_{i}^{*}, \rho_{i+1}^{*}$ and $r_{i}^{*}$, therefore four can be fixed optionally such as

$$
r_{i}^{*}=\frac{r_{\min }+r_{\max }}{2}, \rho_{i}^{*}=\rho_{c r}, v_{i}^{*}=V\left(\rho_{i}^{*}\right), \rho_{i+1}^{*}=\rho_{c r},
$$

and solve equations for the remaining $q_{i-1}^{*}$ and $v_{i-1}^{*}$ terms. Note, we use the same steady-state values for $\rho_{i}(k)$ and $\rho_{i+1}(k)$, i.e. a spatially smooth steady-state situation is chosen.

2) Centering. Introduce the shifted variables as the difference from steady-state values (e.g. $\tilde{\rho}_{i}=\rho_{i}-\rho_{i}^{*}$ ) and rewrite equations (1)-(2) in terms of centered variables and steady-state values.

3) Factorization. Factorize out centered state- and input variables from the obtained nonlinear equations which yield then in an affine form. Bilinear terms could be factorized obviously out, while state variables from non-linear terms are factorized by using the following general formula:

$$
f(x)=A(x) x, A(x)=\int_{0}^{1} \frac{\partial f(\lambda x)}{\partial \lambda x} d \lambda .
$$

Note, this transformation is valid for functions satisfying $f(0)=0$, which is artificially guaranteed by the centering conditions (1). 
Following the main steps of the LPV model transformation, the forthcoming discrete time LPV system $(\Sigma(p))$ representation can be written:

$$
\begin{aligned}
{\left[\begin{array}{c}
\tilde{\rho}_{i}(k+1) \\
\tilde{v}_{i}(k+1)
\end{array}\right] } & =A\left(\tilde{\rho}_{i}(k), \tilde{v}_{i}(k)\right)\left[\begin{array}{c}
\tilde{\rho}_{i}(k) \\
\tilde{v}_{i}(k)
\end{array}\right]+ \\
& +B\left(\tilde{\rho}_{i}(k), \tilde{v}_{i}(k)\right) \tilde{r}_{i}(k)+ \\
& +E_{1}\left(\tilde{\rho}_{i}(k), \tilde{v}_{i}(k)\right)\left[\begin{array}{c}
\tilde{q}_{i-1}(k) \\
\tilde{v}_{i-1}(k)
\end{array}\right]+ \\
& +E_{2}\left(\tilde{\rho}_{i}(k), \tilde{v}_{i}(k)\right)\left[\tilde{\rho}_{i+1}(k)-\tilde{\rho}_{i}(k)\right]
\end{aligned}
$$

In contrast to [5], where an affine scheduling parametrization is proposed, the generic quasi-LPV model is used in the sequel. According to the definition of shock waves, $\left(\tilde{\rho}_{i+1}(k)-\tilde{\rho}_{i}(k)\right)$ term is referred as disturbance input ${ }^{1}$. These shock waves are mathematically represented by discontinues density profiles, with propagation speed depends on the previously defined disturbance term. Note, we do not assume to measure this disturbance term.

\section{B. TP transformation}

Tensor-Product transformation is a numerical method to automatically transform quasi LPV models into a polytopic form by using Higher Order Singular Value Decomposition (HOSVD) technique. For a more exhaustive description of the topic see [8], [9]. The result of the transformation is a finite element polytopic model in the following generic form:

$$
\Sigma(p) \stackrel{T P t}{\longrightarrow} S \bigotimes_{n=1}^{N} w_{n}^{T}\left(p_{n}(k)\right),
$$

where $S$ contains $N$ Linear Time Invariant systems (i.e. the vertecies of the polytope), $w_{n}$ contains a bounded and continuous weighting function $\left(w_{n, i}\left(p_{n}\right)\right)$ and $\bigotimes$ is used as a symbol of multiple product. Moreover, convexity of the description can be ensured by adding the following conditions on the weighting functions to the representation:

$$
\begin{aligned}
\forall n, i, p_{n}(k): & w_{n, i}\left(p_{n}(k)\right) \in[0,1], \\
\forall n, p_{n}(k): & \sum_{i=1}^{I_{n}} w_{n, i}\left(p_{n}(k)\right)=1 .
\end{aligned}
$$

The transformation result in the following description of the freeway dynamics:

$$
\begin{aligned}
x(k+1) & =A(w(k)) x(k)+B(w(k)) u(k)+ \\
& +E_{1}(w(k)) d_{1}(k)+E_{2}(w(k)) d_{2}(k),
\end{aligned}
$$

where the following shorthand notations were introduced:

- $x(k)=\left[\begin{array}{ll}\tilde{\rho}_{i}(k) & \tilde{v}_{i}(k)\end{array}\right]^{T}, \quad u(k)=\tilde{r}_{i}(k)$,

- $d_{1}(k)=\left[\begin{array}{ll}\tilde{q}_{i-1}(k) & \tilde{v}_{i-1}(k)\end{array}\right]^{T}, \quad d_{2}(k)=\tilde{\rho}_{i+1}(k)-$ $\tilde{\rho}_{i}(k)$

- $A(w(k))=\sum_{i=1}^{N} A_{i} w_{i}(k), \quad B(w(k))=\sum_{i=1}^{N} B_{i} w_{i}(k)$,

- $E_{1}(w(k))=\sum_{i=1}^{N} E_{1, i} w_{i}(k), \quad E_{2}(w(k))=$ $\sum_{i=1}^{N} E_{2, i} w_{i}(k)$

with:

$$
0 \leq w_{i}(k) \leq 1, \sum_{i=1}^{N} w_{i}(k)=1
$$

\footnotetext{
${ }^{1}$ Unlike in [5] where only $\tilde{\rho}_{i+1}(k)$ was considered as disturbance and $\tilde{\rho}_{i}(k)$ as a state.
}

After transforming the quasi LPV system to a polytopic LPV representation, algorithm are given to compute set invariance in the sequel.

\section{SET-THEORETIC ALGORITHM}

The algorithm for the analysis of the ramp metering problem is proposed in the sequel. In the sequel, ramp meter input is refered to as control input. Upstream segment's input are denoted as measured inputs, while downstream segment's input is refered as unmeaseured input. Firstly, define the following sets with hyperplane representation ([10]):

- State set: $\mathscr{X}=\mathscr{P}\left(H_{x}, h_{x}\right)=\left\{x: H_{x} x \leq h_{x}\right\}$,

- Input set: $\mathscr{U}=\mathscr{P}\left(H_{u}, h_{u}\right)=\left\{u: H_{u} u \leq h_{u}\right\}$,

- Measured disturbance set: $\mathscr{D}_{1}=\mathscr{P}\left(H_{d_{1}}, h_{d_{1}}\right)=$ $\left\{d_{1}: H_{d_{1}} d_{1} \leq h_{d_{1}}\right\}$,

- Unmeasured disturbance set: $\mathscr{D}_{2}=\mathscr{P}\left(H_{d_{2}}, h_{d_{2}}\right)=$ $\left\{d_{2}: H_{d_{2}} d_{2} \leq h_{d_{2}}\right\}$.

It is assumed that all of these sets are assigned polyhedral sets including the origin as an interior point. Moreover $\mathscr{D}_{1}$ and $\mathscr{D}_{2}$ are assumed to be polyhedral C-set ([10]). Note that these sets can be constructed: the admissible region of the variables are available from detector measurements or from physical considerations. These sets can be shifted according to the steady-state values, which implies the inclusion of the origin.

Secondly, the notion of disturbance invariant set is given, which will play a key role in the algorithm ([10]):

Definition 1: The set $\mathscr{S} \subseteq \mathscr{X}$ is said to be disturbance invariant with respect to the unmeasured signal $d_{2}$ if $\forall x(k) \in$ $\mathscr{S}$ and $\forall d_{1} \in \mathscr{D}_{1}, \exists u \in \mathscr{U}$ such that $x(k+1) \in \mathscr{S}, \forall d_{2} \in \mathscr{D}_{2}$ and $\forall w \in W$.

The interpretation of the above mentioned definition for the ramp metering is as follows: determine the maximal set of measured variables $\left(x, d_{1}\right)$ for which a constrained onramp input $u$ exists keeping the system inside the set in the presence of a shock wave disturbance denoted by $\forall d_{2} \in \mathscr{D}_{2}$.

The outer approximation of the maximal disturbance invariant set is carried out by the following algorithm:

1) Set $t=0, H_{x}^{(t)}=H_{x}, h_{x}^{(t)}=h_{x}$ and set $\mathscr{X}^{(t)}=$ $\mathscr{P}\left(H_{x}^{(t)}, h_{x}^{(t)}\right)$. Fix a tolerance number $\varepsilon>0$ and a maximum number of steps $t_{\max }$.

2) Compute the erosion ([4]) of the set $\mathscr{X}^{(t)}=$ $\mathscr{P}\left(H_{x}^{(t)}, h_{x}^{(t)}\right)$ with respect to the unmeasured disturbance $E_{2}(w) \mathscr{D}_{2}$ :

$$
\begin{aligned}
\overline{\mathscr{P}}\left(H_{x}^{(t)}, \bar{h}_{x}^{(t)}\right)=\left\{x: H_{x}^{(t)}\right. & \left(x+E_{2}(w) d_{2}\right) \leq h_{x}^{(t)}, \\
& \left.\forall d_{2} \in \mathscr{D}_{2}, \forall w \in W\right\},
\end{aligned}
$$

where the $j$-th row of $\bar{h}_{x}^{(t)}$ can be calculated as:

$$
\bar{h}_{x, j}^{(t)}=h_{x, j}^{(t)}-\max _{w} \max _{d_{2} \in \mathscr{D}_{2}} H_{x, j}^{(t)} E_{2}(w) d_{2} .
$$

where index $j$ denotes the $j$ th row of a matrix or the $j$ th element of a vector, depending on the variable it belongs to. 
3) Expand the set $\overline{\mathscr{P}}\left(H_{x}^{(t)}, \bar{h}_{x}^{(t)}\right)$ in the extended statecontrol-measured disturbance space as follows:

$$
\begin{array}{r}
\mathscr{M}^{(t)}=\left\{\left(x, u, d_{1}\right): u \in \mathscr{D}, d_{1} \in \mathscr{D}_{1},\right. \\
A(w) x+B(w) u+E_{1}(w) d_{1} \in \overline{\mathscr{P}}\left(H_{x}^{(t)}, \bar{h}_{x}^{(t)}\right), \\
\forall w \in W\} .
\end{array}
$$

This set can be computed by the following inequalities for $\left(x, u, d_{1}\right)$ :

$$
\begin{aligned}
H_{x}^{(t)}\left[\begin{array}{lll}
A(w) & B(w) & E_{1}(w)
\end{array}\right]\left[\begin{array}{c}
x \\
u \\
d_{1}
\end{array}\right] \leq \bar{h}_{x}^{(t)} \\
H_{u} u \leq h_{u}, \\
H_{d_{1}} d_{1} \leq h_{d_{1}} .
\end{aligned}
$$

4) Compute the projection of the set $\mathscr{M}^{(t)}$ onto the statemeasured disturbance subspace:

$$
\mathscr{R}^{(t)}=\left\{\left(x, d_{1}\right): \exists u, \text { s.t. }\left(x, u, d_{1}\right) \in \mathscr{M}^{(t)}\right\},
$$

with the following half-plane representation:

$$
H_{x, d}\left[\begin{array}{c}
x \\
d_{1}
\end{array}\right] \leq h_{x, d}
$$

5) Calculate the following set:

$$
\mathscr{F}^{(t)}=\left\{x: \forall d_{1} \in \mathscr{D}_{1}, \exists u \text {, s.t. } x(k+1) \in \mathscr{R}^{(t)}\right\},
$$

Since $\mathscr{D}_{1}$ is convex, this set $\left(\mathscr{F}^{(t)}\right)$ can be computed by evaluating the half-plane representation of $\mathscr{R}^{(t)}$ on the vertices.

6) Set:

$$
\mathscr{X}^{(t+1)}=\mathscr{F}^{(t)} \bigcap \mathscr{X}
$$

7) If

$$
\mathscr{X}^{(t)} \subseteq(1+\varepsilon) \mathscr{X}^{(t+1)}
$$

then stop successfully.

8) If $\mathscr{X}^{(t+1)}=\emptyset$ then stop unsuccessfully.

9) If $t>t_{\max }$ then stop indeterminately.

10) Set $k=k+1$ and go to step 2 .

The algorithm is initialized from the complete set of admissible states. First, the effect of unmeasured disturbances is taken into consideration through erosion. Since the disturbance can not steer the trajectory out of the eroded set, it is enough for invariance to find such $\left(x, u, d_{1}\right)$ triplets, which keep the nominal ( $d_{2}$-free) system inside $\mathscr{P}$. This is performed in the expansion step by imposing condition (8). Finally in Step 5. the additional information of measured disturbances is incorporated by selecting states with the complete $\mathscr{D}_{1}$ in the extended $\left(x, d_{1}\right)$ space.

\section{NUMERICAL EXAMPLE}

The section provides a numerical example based on traffic measurements to illustrate the proposed algorithm.

A $\Delta_{i}=500 \mathrm{~m}$ long stretch of freeway A12 in the Netherlands has been chosen as a test case, where a two lane on-ramp is connected to three main lanes. Physical constraints of the on-ramp are $r_{\min }=600 \frac{\mathrm{veh}}{\mathrm{h}}, r_{\max }=2000 \frac{\mathrm{veh}}{\mathrm{h}}$.

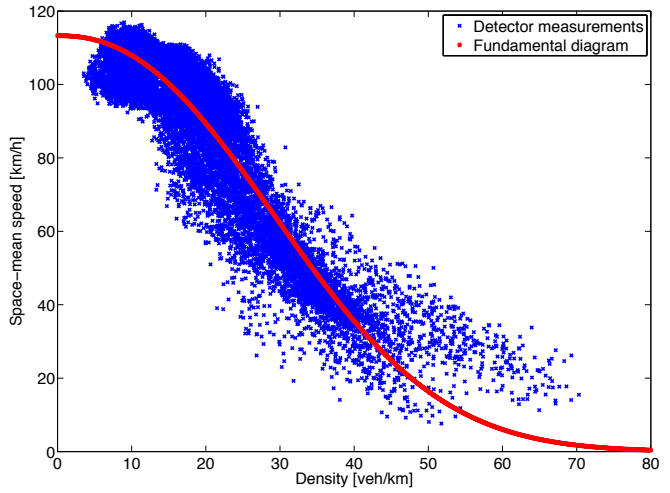

Fig. 2. The fitted fundamental diagram

TABLE I

MODEL PARAMETERS

\begin{tabular}{|c|c||c|c|}
\hline$a$ & 2.2911 & $v$ & $35 \frac{\mathrm{km}^{2}}{\mathrm{~h}}$ \\
\hline$v_{f}$ & $113.2774 \frac{\mathrm{km}}{\mathrm{h}}$ & $\kappa$ & $13 \frac{\mathrm{veh}}{\mathrm{km}}$ \\
\hline$\rho_{c r}$ & $26.1170 \frac{\mathrm{veh}}{\mathrm{km}}$ & $\delta$ & 1.4 \\
\hline$\tau$ & $20 \mathrm{sec}$ & & \\
\hline
\end{tabular}

Measurements of the installed detector have been processed and smoothed to filter measurement noises. Parameters of the fundamental relationship (3) were determined through a non-linear least squares fitting on the measured data set. The fitted diagram can be seen on Figure 2. The values of the remaining parameters were taken from the literature as typical values. Model parameters are summarized in Table I.

Once the model parameters were determined, the steadystate values has been calculated, based on the computation setup presented in Section III-A. The obtained values are summarized in Table II.

Next, a generic exact LPV model was constructed through the introduction of the centered variables. The parameter dependent structure was then transformed into the proposed polytopic form by using TP-tool and a $80 \times 110$ grid in the state-space, in traffic density and in space mean speed. The algorithm resulted in three density-dependent and two spacemean speed-dependent weights (Figure 3), which coincide a number of $3 \times 2=6$ vertecies each represented as LTI system. The HOSVD based polytopic form and the original quasi LPV model have been numerically checked over 2000 random points. The maximum error was found in the range of $10 e-12$ and the root mean square error is in the range $10 e-$ 13 , caused by the numerical computation issue. Therefore we can conclude that the two models(generic and polytopic

TABLE II

MODEL PARAMETERS

\begin{tabular}{|c|c||c|c||c|c|}
\hline$\rho_{i}^{*}$ & $26.1170 \frac{\mathrm{km}}{\mathrm{h}}$ & $q_{i-1}^{*}$ & $4436 \frac{\mathrm{veh}}{\mathrm{h}}$ & $\rho_{i+1}^{*}$ & $26.1170 \frac{\mathrm{km}}{\mathrm{h}}$ \\
\hline$v_{i}^{*}$ & $73.2131 \frac{\mathrm{km}}{\mathrm{h}}$ & $v_{i-1}^{*}$ & $88.7221 \frac{\mathrm{km}}{\mathrm{h}}$ & $r_{i}^{*}$ & $1300 \frac{\mathrm{veh}}{\mathrm{h}}$ \\
\hline
\end{tabular}



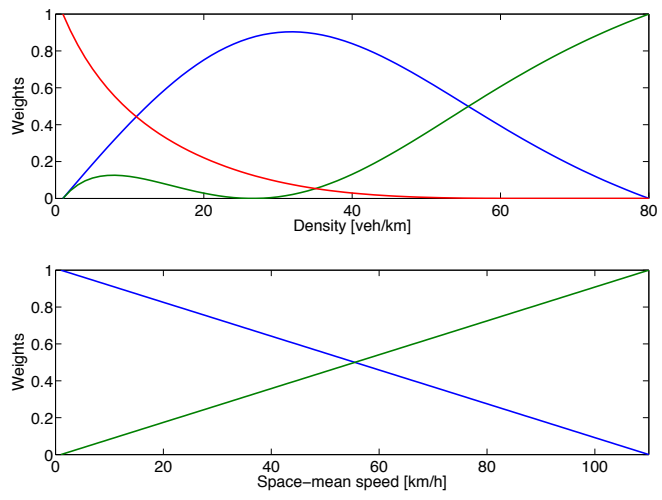

Fig. 3. State-dependent weights of the TP model

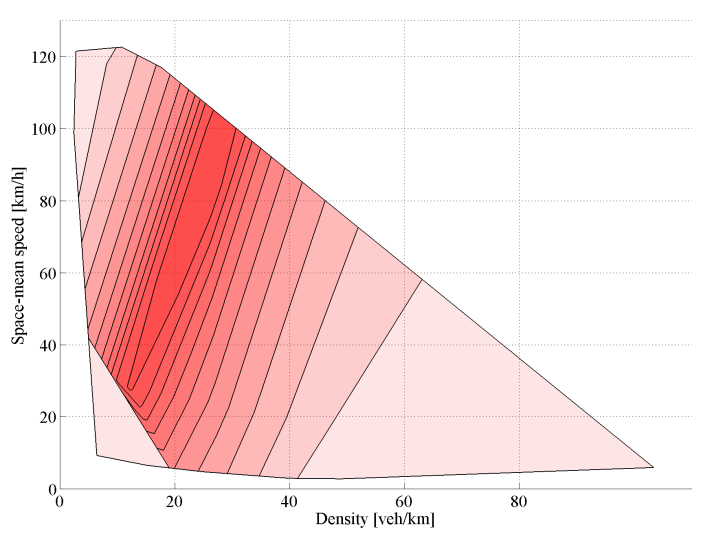

Fig. 4. The iteration steps and the maximal disturbance invariant set of the proposed method

LPV) are numerically equivalent.

Finally, the presented set-theoretic algorithm was applied to the case study. The hyperplane representation of the state space $\mathscr{X}$ was determined by constructing a convex hull based on existing detector measurements. The set of control input $\mathscr{U}$ is given by the centered physical constraints, i.e.: $-700 \leq \tilde{r}_{i} \leq 700$. For the measured and unmeasured disturbances the following traffic scenario was considered. In downstream direction a moderately congested traffic is chosen by the appropriate calibration of the set of unmeasured signal. From upstream direction, the entering flow is characterized to be moderately under the section's maximal capacity. This scenario has the importance from traffic engineering point of view (backward propagation of traffic jams).

The tolerance and the maximal iteration number were set to $\varepsilon=0.05, t_{\max }=100$. The set theoretic algorithm sufficiently converged after 11 iterations. The result are depicted in Figure 4.

Figure 4. shows the computed maximal disturbance invariant set i.e. the set of states which can be kept invariant by constrained ramp metering. These states could be considered as the maximal region of applicability of the ramp metering. The algorithm states that one could only solve the constrained control problem inside the computed final set. The exact effect of the control, i.e. how the closed-loop state trajectory evolves inside this region, can only be shown by minimizing the appropriate control objective (such as the total time spent measure).

Moreover the same traffic scenario was repeated with tighten control constraints. This limited control energy can be considered as a nearly constant uncontrolled on-ramp demand. In this case the algorithm was stopped unsuccessfully, which means that the upstream congestion propagates backward and could lead to traffic breakdown. This result clearly illustrates the importance of freeway ramp metering.

\section{CONCLUSION AND FURTHER RESEARCH}

The paper proposed a set-theoretic analysis of local ramp metering problem in freeways traffic flow context.

Firstly, a well-known second-order and non-linear model of freeway flow segment is transformed into a generic quasiLPV form. Based on parameter-dependent structure, a novel polytopic description is developed by using HOSVD based Tensor-Product transformation, afterwards.

Secondly, set-theoretic algorithm was developed to investigate the effect of constrained ramp-metering on freeways dynamics. The algorithm computes the outer approximation of the maximal disturbance invariant set, based on the developed polytopic LPV form.

Numerical example is given to illustrate the viability of the proposed technique, where model parameter have been obtained through real detector measurements. The example showed how ramp-metering can prevent the backward propagation of traffic jams.

The current description do not consider on-ramp queue dynamics. Taking this effect in the future will introduce a state and disturbance dependent constraint on the control input, and hence will result in a more realistic solution.

\section{ACKNOWLEDGMENTS}

The traffic data are provided by, and used with the permission of Rijkswaterstaat - Centre for Transport and Navigation.

The authors gratefully acknowledge to the support by the New Hungary Development Plan (Project ID: TÁMOP4.2.1/B-09/1/KMR-2010-0002) and by the Hungarian Scientific Research Fund (OTKA) through grant No. CNK 78168 and by János Bólyai Research Scholarship of the Hungarian Academy of Sciences which are gratefully acknowledged.

The authors express their thanks to Andreas Hegyi from Delft University of Technology, Faculty of Civil Engineering, for his valuable and important comments. Special thanks to Aeje.

This work has been supported by Chalmers new initiatives in Transportation, therefore B.Kulcsár acknowledges the support of the Area of Advance in Transportation.

\section{REFERENCES}

[1] M. Papageorgiou and A. Kostialos, "Freeway Ramp Metering: An Overview", IEEE Transactions on Intelligent Transportation Systems, vol. 3., no. 4. 2002, pp. 271-281. 
[2] M. Papageorigiou, H. Hadj-Salem, and J.-M. Blosseville, "ALINEA: a local feedback control law for on-ramp metering", Transportation Research Record, no. 1320, pp. 5864, 1991.

[3] A. Hegyi, B. De Schutter, and H. Hellendoorn, "Model predictive control for optimal coordination of ramp metering and variable speed limits" Transportation Research Part C, vol. 13, no. 3, pp. 185-209, 2005.

[4] M. Papageorgiou, "Some remarks on macroscopic traffic flow modelling" Transportation Research Part A, vol. 32, no. 5, pp. 323-329, 1998.

[5] T. Luspay, B. Kulcsár, I. Varga and J. Bokor, "Parameter-dependent modeling of freeway traffic flow" Transportation Research Part C, vol. 18 , iss. 4, pp. 471-488, 2010.

[6] M. Cremer and M. Papageorgiou, "Parameter identification for a traffic flow model" Automatica, vol. 17, iss. 6, pp. 837-843, 1981.

[7] T. Luspay, B. Kulcsár, J.W. van Wingerden, M. Verhaegen and J. Bokor, "Linear Parameter Varying Identification of Freeway Traffic Models" IEEE Transactions on Control System Technology, vol. 19, no. 1, pp. 31-45, 2011.

[8] P. Baranyi, "TP model transformation as a way to LMI based controller design", IEEE Transaction on Industrial Electronics, vol. 51, no. 2, pp. 387- 400, 2004.

[9] D. Tikk, P. Baranyi, R. J. Patton and J. Tar, ”Approximation Capability of TP model forms", Australian Journal of Intelligent Information Processing Systems, vol. 8., no. 3., pp.155-163, 2004.

[10] F. Blanchini, S Miani, "Set-Theoretic Methods in Control", Birkhuser Boston, 2008. 